\title{
GIT1Y321 phosphorylation is required for ERK1/2- and PDGF-dependent VEGF secretion from osteoblasts to promote angiogenesis and bone healing
}

\author{
ZE RUI $^{1 *}$, XIANG LI $^{1 *}$, JIN FAN $^{1}$, YONGXIN REN $^{1}$, YUFENG YUAN $^{2}$, \\ ZHENGZHE HUA $^{1}$, NING ZHANG ${ }^{1}$ and GUOYONG YIN ${ }^{1}$ \\ ${ }^{1}$ Department of Orthopaedics and ${ }^{2}$ The Central Library of The First Affiliated Hospital, The First \\ Affiliated Hospital of Nanjing Medical University, Jiangsu, Nanjing 210029, P.R. China
}

Received February 7, 2012; Accepted April 9, 2012

DOI: $10.3892 /$ ijmm.2012.1058

\begin{abstract}
Bone healing depends on vascular endothelial growth factor (VEGF) secretion from osteoblasts to promote angiogenesis. We examined the influence of the tyrosine 321 site of $\mathrm{G}$ protein-coupled receptor kinase interacting protein 1 (GIT1) on platelet-derived growth factor (PDGF)-induced VEGF synthesis in vitro and on bone healing in vivo. Cultured osteoblasts were prepared from calvaria of 1-2-day-old rats. The phospho-activation of extracellular signal-regulated kinases 1/2 (ERK1/2), GIT1, the interaction between GIT1 and ERK1/2, and VEGF mRNA expression were measured in response to PDGF. In addition, PDGF was applied following pretreatment with the MEK1/2 inhibitor PD98059 or the Src inhibitor PP2. We mutated tyrosines 293 or 321 of GIT1 individually to phenylalanine (mutants GIT1Y293F and GIT1Y321F) and incorporated these mutants and native GIT1 into lentivirus vectors. The relationship between GIT1 and ERK1/2, and VEGF mRNA expression in cultured osteoblasts were detected after infection with GIT1WT-, GIT1Y293F- and GIT1Y321F-expressing lentivirus in response to PDGF. Bone healing and expression of VEGF and the angiogenic marker PECAM-1 were evaluated after infection at the fracture site. Activation of ERK1/2 by phosphorylation, GIT1 tyrosine phosphorylation, GIT1-ERK1/2 interaction, and VEGF mRNA expression were all significantly increased in osteoblasts after PDGF stimulation, but all responses were dramatically inhibited by pretreatment with PD98059. Tyrosine phosphorylation,
\end{abstract}

Correspondence to: Dr Guoyong Yin, Department of Orthopaedics, The First Affiliated Hospital of Nanjing Medical University, 300 Guang Zhou Road, Jiangsu, Nanjing 210029, P.R. China

E-mail: guoyong_yin2005nanjing@yahoo.com

${ }^{*}$ Contributed equally

Key words: G protein-coupled receptor kinase interacting protein 1, vascular endothelial growth factor, extracellular signal-regulated kinases $1 / 2$, bone healing
GIT1 interaction with ERK1/2, and VEGF mRNA expression were dramatically inhibited by pretreatment with PP2 or infection with GIT1Y321F-expressing lentivirus. Expression of VEGF and PECAM-1 was significantly lower at the fracture sites infected with GIT1Y321F-expressing lentivirus and bone healing was significantly delayed compared to fracture sites infected with GIT1WT. In conclusion, tyrosine 321 of GIT1 is a critical phosphorylation site for GIT1 interaction with ERK1/2, regulation of ERK1/2 activation, VEGF expression and angiogenesis at the fracture site. Furthermore, GIT1 is a seminal signaling protein regulating bone healing.

\section{Introduction}

There is a close relationship between bone formation and angiogenesis. The germinal center of the bone is located around blood vessels during embryonic osteogenesis. During development and elongation of the bone, angiogenesis factors in the epiphyseal growth plate induce blood vessel growth into the metaphysis and cartilage region, thus promoting the formation of new bone. In addition, angiogenesis is required for endochondral ossification during fracture healing (1). In this process, avascular cartilage tissue is gradually replaced by bone tissue. Many factors influence fracture healing by affecting angiogenesis at the fracture site, including fibroblast growth factor (FGF), transforming growth factor (TGF), platelet-derived growth factor (PDGF), prostaglandin $\mathrm{E}$ (PGE), vascular endothelial growth factor (VEGF), and tumor necrosis factor (TNF). Of these factors, VEGF is particularly important in angiogenesis, acting on vascular endothelial cells to promote proliferation and angiogenesis $(2,3)$. Various types of cells secrete VEGF, including fibroblasts, smooth muscle cells, hypertrophic chondrocytes and osteoblasts. Hypertrophic chondrocytes secrete VEGF, and induced metaphysis blood vessel growth into the cartilage region, thus promoting ossification. Secretion of VEGF from osteoblasts is critical for bone metabolism, and osteoblasts secrete VEGF during fracture healing (3).

Despite the importance of osteoblast VEGF release to long bone development and fracture healing, the molecular signal transduction mechanisms for the induction and control 
of VEGF release are unknown. VEGF release is induced by many factors in vivo, including hypoxia, steroids and cytokines like insulin-like growth factor-1 (IGF-1), keratinocyte growth factor, epidermal growth factor (EGF), TNF- $\alpha$, TGF, interleukin-1 (IL-1), IL-6. Intracellular signaling cascades involving tyrosine kinases also greatly impact VEGF release and signal transduction. Extracellular signal-regulated kinases $1 / 2$ (ERK1/2) play a central role in regulating VEGF secretion (4). First, ERK1/2 signals directly to the VFGF promoter to control expression of VEGF protein under normoxia. Under hypoxia, hypoxia inducible factor-1 (HIF-1) is activated by ERK1/2 to promote the expression of VEGF protein. ERK1/2 is a serine and threonine kinase that interacts with a myriad of signaling pathways (5). In turn, many signaling molecules regulate ERK1/2 activity, including activator protein 1 (AP-1), angiotensin-II (Ang-II), and negative regulatory factor (Nef) (6).

We previously reported that $\mathrm{G}$ protein-coupled receptor kinase interacting protein 1 (GIT1) is an important regulator of ERK1/2 activity by acting as a specific shuttle protein that associates ERK1/2 with upstream kinases and downstream targets (7). We further demonstrated that GIT1 knockout mice had both greatly reduced ERK1/2 activity and VEGF expression. In addition, GIT1 knockout impeded formation of the rat pulmonary artery, indicating that the protein is involved in angiogenesis (8). The yeast two-hybrid method demonstrated that GIT1 can combine with $\mathrm{G}$ protein coupled receptor kinase 2 (GRK2). This interaction regulates cytoskeletal structure, membrane transport, other signaling molecules, and cell polarity (9). While GIT1 itself has no catalytic activity, GIT1 activation by tyrosine phosphorylation triggers a series of intracellular signal transduction events. We have previously reported that GIT1 was tyrosine phosphorylated by Src, leading to increased ERK1/2 activity (10). Indeed, protein tyrosine phosphorylation is vital for GIT1 signaling. Webb et al (11) described several important tyrosine phosphorylation sites in GIT1 by mass spectrometry analysis. We also found that GIT1 Y392 was phosphorylated by Src, and that Src-mediated tyrosine phosphorylation activated intracellular PLC $-\gamma$, leading to podosome formation in smooth muscle cells (12). Phosphorylation of GIT1Y321 is key to enhancing ERK1/2 activity $(7,13)$.

In this study, we clarified the mechanisms of GIT1Y321 phosphorylation during PDGF-induced secretion of VEGF from osteoblasts. We then examined the role of this signaling cascade in bone fracture healing.

\section{Materials and methods}

Materials and reagents. Twenty 1-2-day-old Sprague Dawley (SD) rats (both male and female) and $30 \mathrm{SD}$ male rats weighing about 200-300 g were obtained from the Department of Animal Science, Nanjing Medical University. The drugs and reagents (and their suppliers) were as follows: Dulbecco's modified Eagle's medium (DMEM) and fetal bovine serum (FBS) were from Gibco (USA); EDTA-trypsin, collagenase II, and Src inhibitor PP2 and MEK inhibitor PD98059 were from Sigma (USA); GTI1 antibody, Flag antibody, PECAM-1 antibody were purchased from Cell Signaling Technology (USA) while the ERK1/2 antibody, pERK1/2 antibody, pERK2 antibody, and VEGF antibody were purchased from Santa Cruz Biotechnology, Inc. (USA); tubulin antibody and 4G10 anti- body were from Cell Signaling (USA). Finally, VEGF primers were custom synthesized by Invitrogen (USA).

Lentivirus grain package and titration. Various restriction enzymes, T4 ligase, pyrobest DNA polymerase, and a DNA fragment purification kit were purchased from Gibco. A sitedirected mutagenesis kit was purchased from Takara Bio, Inc. (Japan). A mouse testis cDNA library, lentiviral vector plasmids pLJM-GFP, lentiviral packaging plasmids Delta891, pVSVG, and 293T cell packaging virus were from the Laboratory of Cell Biology, Nanjing Medical University. The pCMV-Tag2B-GIT1WT, pCMV-Tag2B-GIT1Y293F, pCMVTag2B-GIT1Y321F and pCMV-Tag2B-GIT1Y392F vectors were a kind gift of Professor Bradford C. Berk (University of Rochester, NY). The GIT1, GIT1Y293F and GIT1Y321F vectors were constructed into pLJM to form pLJM-GIT1, pLJM-GIT1Y293F and pLJM-GIT1Y321F. These plasmids and supporting original viral packaging (delta891 and pVSVG) were transfected into $293 \mathrm{~T}$ cells. The original medium was replaced by complete medium after $6 \mathrm{~h}$ transfection and was incubated for $48 \mathrm{~h}$ to allow for viral replication. The supernatant containing lentiviral particles was collected and concentrated. The virus titer was determined by a previously described method and stored at $-80^{\circ} \mathrm{C}$ (14).

Isolation and culture of osteoblasts and group. Primary cultures of neonatal rat calvarial (NRC) cells were obtained from the calvaria of 1-2-day-old SD rat pups by the method described by Frick and Bushinsky with some modifications (15). All procedures were performed in accordance with Nanjing Medical University Animal Care Committee guidelines. Briefly, neonatal rats were sacrificed by $75 \%$ ethanol soak for $20 \mathrm{~min}$, and the calvaria were immediately dissected and placed in chilled DMEM. The calvaria were cleaned of all loose tissues, including the periosteum and dura mater, and washed in a dilute solution of phosphate-buffered saline (PBS). Cells were then released by five sequential 10-min digestions with buffer containing $0.1 \%$ collagenase and $0.2 \%$ hyaluronidase. Fractions 2-5 were collected, pelleted by centrifugation at 1,500 rpm for $3 \mathrm{~min}$, resuspended in DMEM, and plated in T-75 flasks. Cells were grown in DMEM supplemented with $10 \% \mathrm{FBS}, 100 \mathrm{IU} / \mathrm{ml}$ penicillin, and $100 \mathrm{IU} / \mathrm{ml}$ streptomycin and incubated at $37^{\circ} \mathrm{C}$ in a $5 \% \mathrm{CO}_{2}$ atmosphere. Confluent cells were passaged in medium with $0.25 \%$ trypsin and EDTA and plated onto petri dishes and allowed to grow to confluence. Culture plates were divided into a non-pretreated group and pretreated group. Osteoblast cultures in the non-pretreated group were transfected with different virus solutions, starved for $6 \mathrm{~h}$ in basal medium, then stimulated for 0 (baseline), $0.5,2,6$ or $8 \mathrm{~h}$ with $10 \mathrm{ng} / \mathrm{ml}$ PDGF. Osteoblast cultures in the pretreated group were starved $6 \mathrm{~h}$ after infection, then pretreated with the MEK inhibitor PD98059 $(10 \mu \mathrm{M})$ or the Src inhibitor PP2 $(10 \mu \mathrm{M})$ for $30 \mathrm{~min}$, followed by PDGF treatment at the same concentration and treatment durations as in the non-pretreated group. Following PDGF treatment, cells were harvested for detection of protein and mRNA expression.

Western blot analysis assays for expression of pERK1/2, ERK1/2, GIT1 and tyrosine phosphorylation GIT1 in osteoblasts, VEGF and GIT1 from callus tissue. Cells from each 
treatment group were lysed and proteins were isolated. Protein concentrations were determined by the BCA protein assay. Separated proteins were transferred to polyvinylidene fluoride (PVDF) membranes at $100 \mathrm{~V}$. Membranes were blocked for $2 \mathrm{~h}$ in $5 \%$ bovine serum albumin (BSA) in PBS and then incubated overnight at $4^{\circ} \mathrm{C}$ in primary antibody. Antibody-treated membranes were washed and incubated in the appropriate secondary antibody $(1: 5,000)$ at $37^{\circ} \mathrm{C}$ for $1 \mathrm{~h}$. Antibody staining was visualized using enhanced chemiluminescence.

Immunoprecipitation assays to detect the association of GIT1 and GIT1Y321F to ERK1/2. Protein samples (100 $\mu \mathrm{g})$ from each group were mixed with $1 \mu \mathrm{g}$ anti-Flag antibody and incubated overnight at $4{ }^{\circ} \mathrm{C}$ with slow shaking. Protein G/A agarose beads were added and the mixture incubated for $2 \mathrm{~h}$ at $4^{\circ} \mathrm{C}$. The mixture was centrifuged at 5,000 rpm for $3 \mathrm{~min}$ at $4^{\circ} \mathrm{C}$. The supernatant was discarded and the beads washed in $1 \mathrm{ml} \mathrm{PBS}$, then centrifuged again at 5,000 rpm for $3 \mathrm{~min}$ at $4^{\circ} \mathrm{C}$. Washing was repeated two more times. Sufficient sample buffer was added in the precipitate followed by boiling at $100^{\circ} \mathrm{C}$ for $5 \mathrm{~min}$. The beads were then centrifuged at $5,000 \mathrm{rpm}$ for $5 \mathrm{~min}$ at $4^{\circ} \mathrm{C}$. After cooling, the supernatant was loaded onto a gel for electrophoretic separation and western blotting as described above.

Bone fracture modeling in rats. Rats (200-300 g) were injected intraperitoneally with $10 \%$ chloral hydrate. The dorsal side of one leg was shaved and sterilized with an iodine solution. An open fracture was created in the tibia using a modification of the method (16). Thirty rats with tibia fractures were divided randomly into three groups of 10 . The first group was injected with GIT1WT lentivirus at the fracture site, the second group was injected with mutant GIT1Y293F virus, and the third group was injected with mutant GIT1Y321F virus at the fracture site. The three groups were housed separately during recovery without any limitation on activity. Starting at 1-3 days after surgery, rats were treated daily with 4,000 M gentamicin i.m. to prevent infection. Rats were sacrificed at $5,7,10$ and 14 days after fracture. The tibia fracture site was $\mathrm{X}$-rayed before sacrificing the animals. Following sacrifice, we examined the range of calli derived and randomly selected rats for immunohistochemical analysis and western blotting.

$R T-P C R$. Callus at the site of fracture were collected to extract total-RNA for amplification. Total-RNA was amplified by two pairs of primers: sense, 5'-TGCGATGCGGGGGCTGC-3' and antisense, 5'-TTTCCTGGTGAGAGTCT-3'. The PCR reaction was run in a $50 \mu 1$ volume. The thermocycle settings used were 36 cycles of $94^{\circ} \mathrm{C}$ for $30 \mathrm{sec}, 55^{\circ} \mathrm{C}$ for $30 \mathrm{sec}$, and $72^{\circ} \mathrm{C}$ for $30 \mathrm{sec}$. After the reaction, $4 \mu \mathrm{l}$ of the reaction solution was combined with $1 \mu \mathrm{l}$ bromophenol blue loading buffer and run on $1.5 \%$ agarose gels $(85 \mathrm{~V})$ and bands were analyzed using a UVP GDS 8000 gel imaging analysis system camera (UVP, Cambridge, UK).

Immunohistochemistry. Tissue samples from the fracture site were isolated at various times following the injury and consecutive paraffin sections were prepared. For immunohistochemical staining, sections were first dewaxed in xylene. Dewaxed sections were soaked for $10 \mathrm{~min}$ in $3 \% \mathrm{H}_{2} \mathrm{O}_{2}$ solution in methanol to eliminate endogenous catalase. Sections were then washed 3 times with PBS wash, treated with $10 \%$ goat serum for $10 \mathrm{~min}$ and incubated in rat anti-mouse VEGF antibody overnight in a wet box. Labeled sections were washed 3 times in PBS ( 2 min each), treated with anti-rat biotinylated secondary antibody for $10 \mathrm{~min}$, washed as before in PBS, incubated in HRP-streptavidin for $10 \mathrm{~min}$, washed in PBS, and finally treated with DAB color liquid at ambient temperature. For quantification, we first selected the middle section of the callus. Multiple sections were used to count the number of VEGF-positive cells under light microscopy.

Immunofluorescence detection of PECAM-1 expression in callus tissue. The methods for PECAM-1 staining were as above except that the primary antibody (anti-PECAM-1) was labeled with a rhodamine-conjugated secondary goat antimouse antibody for $1 \mathrm{~h}$ at $37^{\circ} \mathrm{C}$. Sections were counterstained with DAPI nuclear stain for 5-10 min and mounted in $80 \%$ glycerol/PBS. Rhodamine and DAPI fluorescence were imaged using a Nikon 80i fluorescence microscope. Photomicrographs were processed using Photoshop CS.

Statistical methods. The SPSS 11.0 statistical package was used for all statistical analysis. Measures are expressed as means \pm SD and analyzed by ANOVA with $\mathrm{P}<0.05$ considered statistically significant.

\section{Results}

PDGF increased ERK1/2 activation, GIT1 phosphorylation, the association of GIT1 with ERK1/2, and VEGF mRNA expression. Our previous studies revealed that EGF, angiotensin II, and VEGF stimulate different cell types to activate ERK1/2 activity, increase tyrosine phosphorylation of GIT1, and promote protein-protein interactions between GIT1 and ERK1/2. In this study, osteoblasts were treated with PDGF (10 ng/ml) and ERK1/2 activation, VEGF mRNA expression, and GIT1-ERK1/2 interaction were examined. Indeed, ERK1/2 activation (pERK1/2) was significantly increased after only $0.5 \mathrm{~h}$ exposure to PDGF but decreased with longer stimulation times (Fig. 1A). In contrast, PDGF increased GIT1 tyrosine phosphorylation in osteoblasts and this response was observed for PDGF treatments up to $6 \mathrm{~h}$ and began to decline thereafter) (Fig. 1B). In osteoblasts treated with PDGF for $0.5 \mathrm{~h}$, GIT1 binding to ERK1/2 was enhanced significantly, and like ERK1/2 phosphorylation, decreased back to baseline as the PDGF treatment duration was extended (Fig. 1C). The expression of VEGF mRNA in osteoblasts increased during PDGF stimulation, peaking at $8 \mathrm{~h}$ (the longest treatment time assessed) (Fig. 1D). To clarify the relationship between ERK1/2 activation, GIT1 phosphorylation, GIT1-ERK binding, and VEGF mRNA expression, we performed these same western blotting experiments on lysates from osteoblasts pretreated with the ERK pathway inhibitor PD98059 and the Src inhibitor PP2. Pretreatment with PD98059 inhibited PDGF-induced ERK1/2 activity and simultaneously inhibited expression of VEGF mRNA in osteoblasts (Fig. 2A and D). Pretreatment with PP2 inhibited PDGF-induced tyrosine phosphorylation of GIT1 (Fig. 2B), the interaction of GIT1 with ERK1/2 (Fig. 2C), and significantly reduced VEGF mRNA expression in osteoblasts 


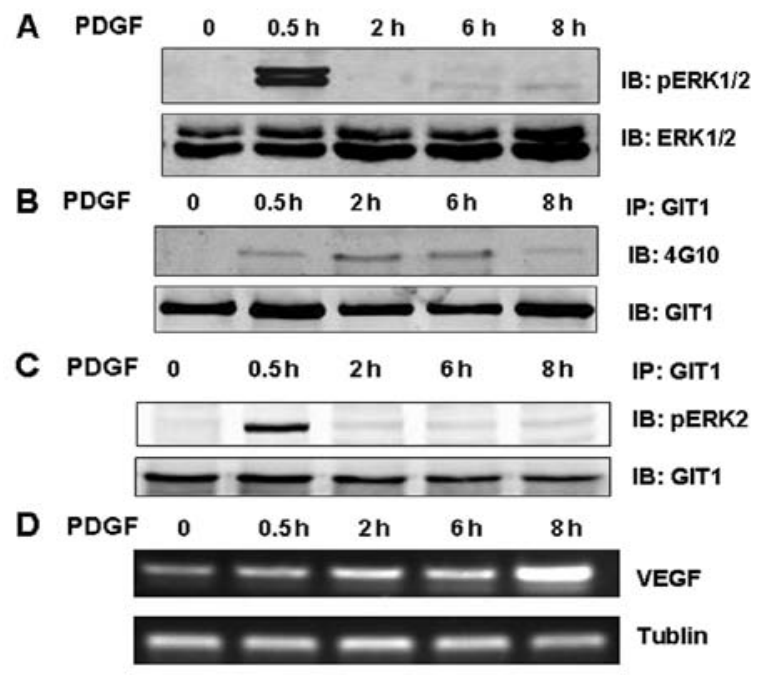

Figure 1. Phospho-activation of ERK1/2 (pERK1/2), tyrosine phosphorylation of GIT1, GIT1-ERK1/2 interaction, and VEGF mRNA expression increased in osteoblasts after PDGF stimulation. (A) Western blot analysis showing ERK1/2 activity at different time points after PDGF stimulation. ERK1/2 activity was significantly increased after $0.5 \mathrm{~h}$ of PDGF stimulation as evidenced by increased pERK1/2 immunoreactivity, but decreased with continued PDGF stimulation. (B) Western blot analysis of GIT1 tyrosine phosphorylation in osteoblasts in response to different PDGF stimulus durations. GIT1 tyrosine phosphorylation was enhanced by $0.5 \mathrm{~h}$ PDGF stimulation but decreased as exposure was increased to $6 \mathrm{~h}$. At $8 \mathrm{~h}$, GIT1 tyrosine phosphorylation was not significantly higher than baseline. (C) The interaction of GIT1 with ERK1/2 was analyzed by immunoprecipitation of ERK1/2 with flag-tagged GIT1. Treatment of osteoblasts with PDGF for $0.5 \mathrm{~h}$ significantly increased the pull-down of ERK1/2, but this GIT1-ERK1/2 interaction decreased with longer PDGF exposure times. (D) VEGF mRNA expression as analyzed by RT-PCR. The expression of VEGF mRNA gradually increased with PDGF exposure time.

(Fig. 2D). These results indicate that ERK1/2 phospho-activation, GIT1 phosphorylation, GIT1-ERK binding, and VEGF mRNA expression in response to PDGF depend on Src and MEK1/2 activation.

Interaction of GIT1 with ERK1/2 and VEGF expression in osteoblasts depends on GIT1 tyrosine 321 phosphorylation. Our previous studies demonstrated that tyrosine phosphorylation of GIT1 altered ERK1/2 activity and that GIT1 tyrosine 321 played an important role. To further clarify the role of Y321 phosphorylation in GIT1-ERK1/2 interaction and VEGF mRNA expression, we infected osteoblasts with mutant or wild type GIT1 and analyzed the responses to PDGF stimulation. We constructed lentiviral vectors including GIT1WT, pCMV-Tag2B-GIT1Y293F, pCMV-Tag2B-GIT1Y321F and pCMV-Tag2B-GIT1Y392F. In osteoblasts treated with PDGF, infection with GIT1WT, GIT1Y293F and GIT1Y392F significantly increased tyrosine phosphorylation, while phosphorylation was completely inhibited in cells infected with a virus containing GIT1Y321F (Fig. 3A). This suggests that Y321 is the target of PDGF-mediated phosphorylation. Furthermore, immunoprecipitation demonstrated that GIT1Y321F inhibited the interaction of GIT1 with ERK1/2, and that the PDGFinduced increase in pERK1/2 activity was effectively blocked in osteoblasts expressing mutant GIT1Y321F (Fig. 3B and C). These results indicate that GIT1Y321 is the key tyrosine phosphorylation site that regulates both the interaction between

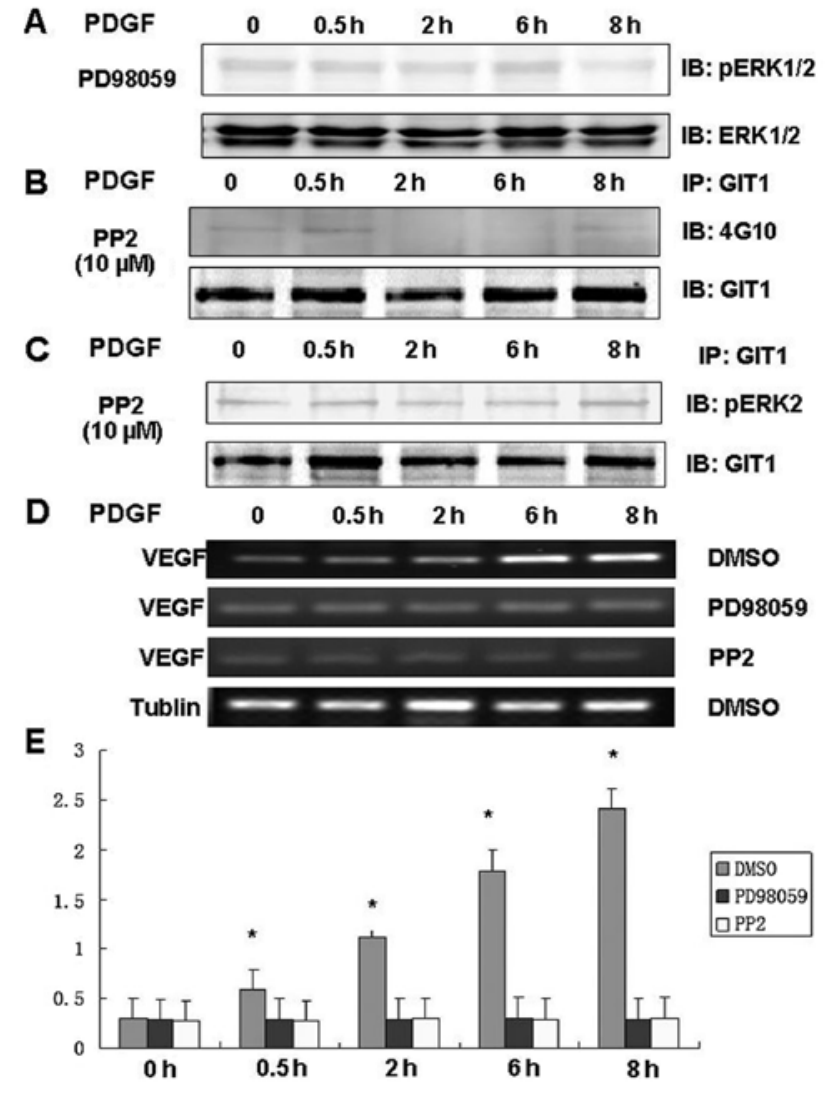

Figure 2. Inhibition of PDGF-mediated enhancement of VEGF mRNA expression, ERK1/2 activity, tyrosine phosphorylation of GIT1, and GIT1-ERK1/2 interaction in osteoblasts by PD 98059 and PP2. (A) Western blot analyses showing that ERK1/2 activity were dramatically inhibited by pretreatment with PD98059. (B) Western blot analysis showing that tyrosine phosphorylation of GIT1 was dramatically inhibited by pretreatment with PP2. (C) Immunoprecipitation of ERK1/2 revealed that the interaction of GIT1 with ERK1/2 was dramatically inhibited by pretreatment with PP2. (D) The expression of VEGF mRNA was dramatically inhibited by pretreatment with PP2 and PD98059. (E) Quantification of VEGF mRNA expression and statistical analysis $\left({ }^{*} \mathrm{P}<0.05\right)$.

ERK1/2 and GIT1 and PDGF-induced ERK1/2 activity. In order to determine the role of Y321 phosphorylation in VEGF mRNA expression, we treated osteoblasts expressing GIT1Y293F and GIT1Y321F with PDGF and analyzed VEGF mRNA expression. Infection with GIT1Y293F had no effect on PDGF-induced VEGF mRNA expression, while GIT1Y321F infection significantly inhibited VEGF mRNA expression in osteoblasts (Fig. 3D). Thus, Y321 is the key phosphorylation site mediating PDGF-induced ERK1/2 activity, ERK1/2-GIT1 interactions and VEGF mRNA expression in osteoblasts.

Phosphorylation of GIT1Y321 regulates VEGF expression, angiogenesis, and healing at the fracture callus. We demonstrated that PDFG-induced VEGF expression in cultured osteoblasts depended on phosphorylation of GIT1Y321, ERK1/2 activation, and GIT1-ERK1/2 interaction. VEGF acts on endothelial cells to trigger angiogenesis, a process that is critical from ossification and fracture healing. We examined the effects of GIT1WT, GIT1Y293F and GIT1Y321F lentiviral infection in a rat tibia fracture model. A group of rats were anesthetized and injured by a single fracture to the tibia. One group was injected with a lentivirus containing GIT1WT and the others with GIT1Y293F and GIT1Y321F lentivirus 
A PDGF (0.5h)

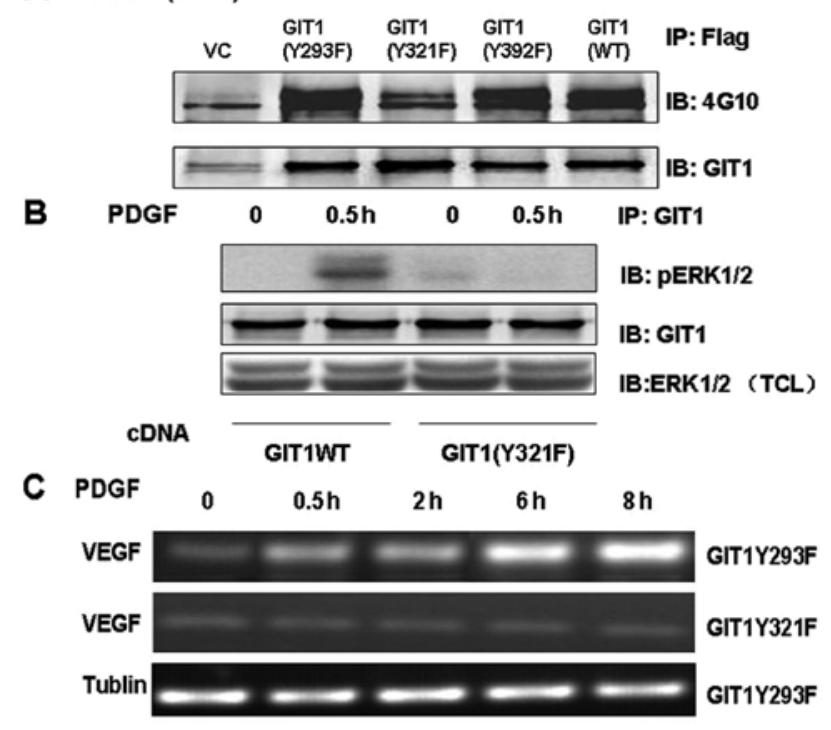

D

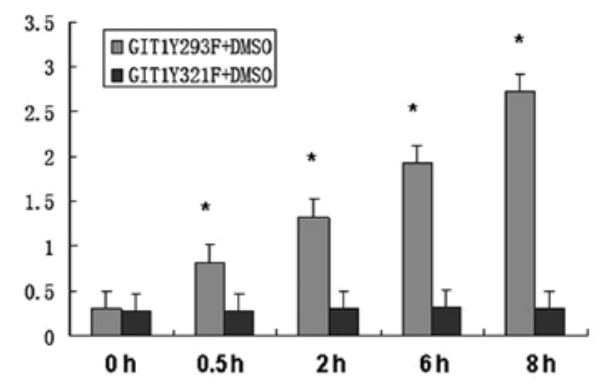

Figure 3. PDGF-mediated enhancement of VEGF mRNA expression, ERK1/2 activity, tyrosine phosphorylation of GIT1, and GIT1-ERK1/2 interaction were all markedly inhibited in osteoblasts infected with GIT1Y321F. (A) Tyrosine phosphorylation of GIT1 was analyzed by immunoprecipitation. PDGF-evoked GIT1 tyrosine phosphorylation significantly increased after infection with GIT1WT, GIT1Y293F and GIT1Y392F lentivirus, but was markedly lower in osteoblasts infected with GIT1Y321F lentivirus. (B) The PDGF-induced interaction of GIT1 with ERK1/2 was dramatically lower in osteoblasts infected with GIT1Y321F lentivirus than in cells infected with GIT1WT. Similarly, enhanced ERK1/2 activity induced by PDGF was effectively blocked in osteoblasts infected with GIT1Y321F. (C) Expression of VEGF mRNA was dramatically lower following PDGF in osteorblasts infected with GIT1Y321F lentivirus than PDGF-treated cells infected with GIT1WT. (D) Statistical results from (C) ( $\left.{ }^{*} \mathrm{P}<0.05\right)$.

respectively. VEGF expression was determined at the fracture site (Fig. 4A). At 5, 7, 10 and 14 days after lentiviral infection, we detected VEGF protein expression. Expression of VEGF was significantly elevated at $5,7,10$ and 14 days after infection with GIT1WT and GIT1Y293F mutants, while VEGF expression in GIT1Y321F lentiviral infected bone was barely above baseline (Fig. 4B and C). To confirm that VEGF protein expression was inhibited by GIT1Y321F infection, callus sections were stained by immunohistochemistry. As shown in Fig. 5A, there was significantly higher VEGF immunoreactivity in calli sections infected with GIT1WT lentivirus compared to GIT1Y321F lentivirus in 7 and 14 days. VEGF is a major inducer of angiogenesis, so reduced expression would tend to suppress angiogenesis. To confirm that reduced VEGF expression did indeed result in lower angiogenesis at the fracture site, we stained calli sections with an antibody against the angiogenic marker protein PECAM-1. As shown in Fig. 5B, 7 and 14 days after fracture, angiogenesis was significantly
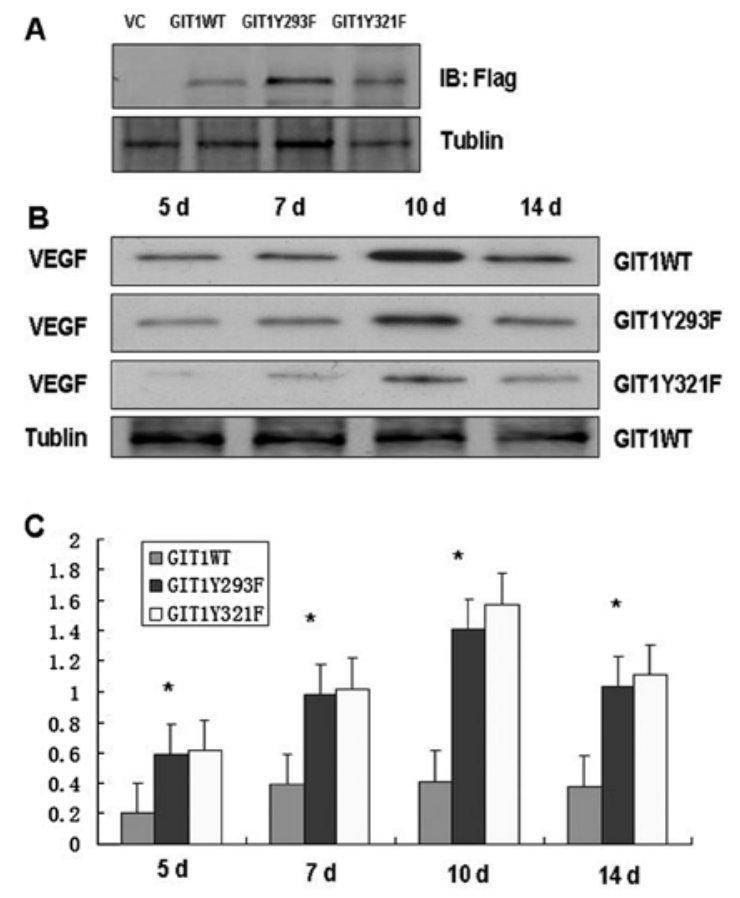

Figure 4. Bone tissue infected with GIT1Y321F shows reduced expression of VEGF and PECAM-1 proteins at the fracture site compared to fractures infected with GIT1WT. (A) Western blotting with anti-Flag 7 day after GIT1WT, GIT1Y293F and GIT1Y321F lentiviral infection showing expression of proteins. (B) Western blot analyses from calli samples 5, 7, 10 and 14 days after fracture. Tissue infected with GIT1Y321F lentivirus showed dramatically lower VEGF protein expression compared to fracture sites infected with lentivirus containing GIT1WT or GIT1Y293F. (C) Densitometric and statistical analysis of western blot analyses shown in (B) ( $\mathrm{P}<0.05)$.

lower at fracture sites injected with GIT1Y321F lentiviral than at sites injected with GIT1WT lentivirus. Previous studies demonstrated that reduced angiogenesis will impede fracture healing. As predicted the healing of the tibial fracture was greatly reduced on Day 14 by GIT1Y321F lentiviral infection (Fig. 5D). In contrast, fractures infected with GIT1WT were fully healed on Day 14, indicating that tyrosine 321 phosphorylation is critical for bone healing by upregulating the expression of the angiogenic factor VEGF.

\section{Discussion}

Fracture healing is a complex physiological process involving the coordinated expression of immune, angiogenic, and ossification factors. VEGF is a disulfide-linked homodimer glycoprotein that promotes endothelial cell proliferation and angiogenesis during development. At a fracture site, VEGF also promotes vascular permeability, cartilage cell differentiation, osteoblast differentiation, and osteoclast recruitment, all vital processes for new bone formation (17). Thus, VEGF is a major regulator of fracture healing. Promotion of angiogenesis facilitates bone healing, while VEGF indirectly induces osteoblast proliferation, migration, and differentiation by stimulating endothelial cells to secrete several osteoanabolic factors, including endothelin-I and insulin-like growth factor-I (17). Vascularization by VEGF promotes cartilage absorption, ossification, and subperiosteal bone formation (18). Niida et al (19) have shown that VEGF promotes osteoclast 
A

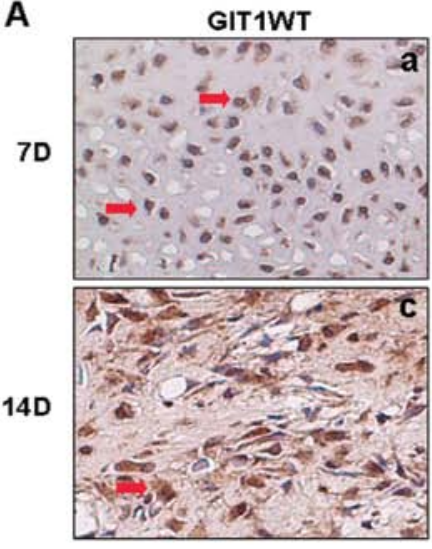

B
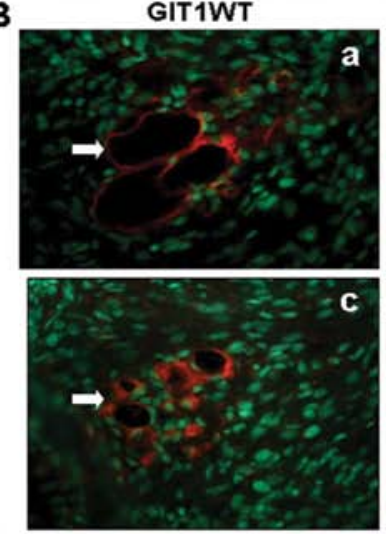

C

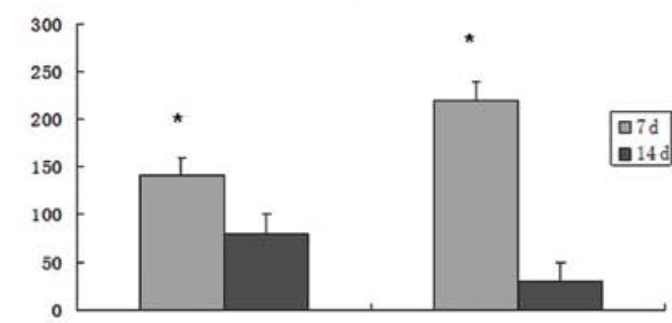

D

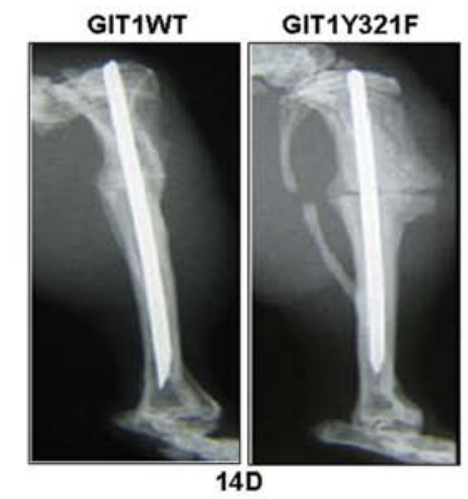

Figure 5. Infection with GIT1 (Y321F) reduces angiogenesis and fracture healing. (A) Histochemical staining of callus 7 and 14 days after fracture revealed more intense VEGF protein immunoreactive staining in sections from tissue infected with GIT1WT than in tissue infected with GIT1Y321F lentivirus (red arrow shows one of VEGF positive staining cells). (B) Fluorescence micrographs of callus 7 and 14 days after fracture and stained for the angiogenesis marker PECAM-1 (rhodamine-red) and counterstained with DAPI (green). Tissue sections infected with GIT1WT showed significantly higher PECAM-1 expression than tissue infected with GIT1Y321F lentivirus (white arrow shows one of PECAM-1 positive stain). (C) Quantification of small vessel number showing higher number in callus infected with GIT1WT compared to GIT1Y321F ( $\mathrm{P}<0.05)$. (D) X-ray of rat tibia 14 days after GIT1Y321F lentiviral infection showing that the fracture had yet to heal, while a similar X-ray from a tibia infected with GIT1WT revealed complete healing of the fracture. differentiation and bone resorption similar to that evoked by macrophage colony-stimulating factor (M-CSF).

VEGF is a multifunctional cytokine secreted by platelets, megakaryocytes, endothelial cells, osteoblasts, and tumor cells in response to a multitude of extracellular signals. Deckers et al (20) confirmed that proliferation of osteoblasts could regulate VEGF release and that bone morphogenic proteins stimulated osteoblasts to secrete VEGF. Induction of VEGF by IGF-I in osteoblast-like cells was mediated by the PI3K signaling pathway. Transforming growth factor- $\beta$ (TGF- $\beta$ ) stimulated heat shock protein 27 (HSP27) induction via $\mathrm{p} 44 / \mathrm{p} 42$ mitogen-activated protein (MAP) kinase, p38 MAP kinase and stress-activated protein kinase/c-Jun $\mathrm{N}$-terminal kinase in osteoblast-like MC3T3-E1 cells, and that the release of VEGF was induced by TGF- $\beta$ in these cells (21). Stimulation of EGF receptors regulated the production of VEGF through the ERK1/2 pathway (22). Application of the ERK1/2 inhibitor 6-thioguanine inhibited the secretion of VEGF, as did application of the MEK1 inhibitor PD98059 (23), underscoring the importance of MEK1/ERK1/2 signaling in the regulation of VEGF. The VEGF promoter region contains binding sites for the transcription factor Sp1, and Sp1 activity is controlled by extracellular signals through ERK1/2. Indeed, ERK1/2 activity and phosphorylation are correlated with Sp1 activity to regulate the expression of VEGF (24).

We have previously reported that GIT1 regulates ERK1/2 activity through direct protein-protein interactions, likely by acting as a specific shuttle protein in the MEK1/ERK1/2 signaling pathway (25). The GIT1 protein is an important signaling protein in osteoblasts. The interaction of GIT1 with signaling molecules is mediated by multiple interacting domains, including an N-terminal ARFGAP domain, an ankyrin repeats (ANK) domain, a PIX-binding domain (Spa2 homology domain 1, SHD) and a C-terminal paxillin-binding domain (26). The ARF-GAP domain of GIT1 regulates ARF1, ARF5, ARF6 and other signaling molecules to control formation of vesicles from the Golgi complex, in addition to roles in endosome-mediated intermembrane and membrane transport, cell proliferation and migration, and synaptogenesis (27). At the SHD domain of GIT1, a complex set of protein interactions occurs to form large oligomeric protein complexes with a multitude of functions. The main binding partner for this domain is $\mathrm{p} 21$-activated kinase interacting exchange factor (PIX). We have previously reported that GIT1, through the SHD domain, allows the GIT1 CC2 domain to combine with MEK1 and ERK1/2 to facilitate ERK1/2 activation (28). We also found that GIT1 was tyrosine phosphorylated by Src, while the Src inhibitor PP2 inhibited tyrosine phosphorylation of GIT1 in osteoblasts in vitro $(7,13)$.

Tyrosine phosphorylation is essential to GIT1 function. Mass spectrometry found that the SHD and CC2 regions have three major tyrosine phosphorylation sites at 293, 321 and 392 (11). Segura et al (29) demonstrated that phosphorylation at GIT1 tyrosine 392 could promote protein-protein interaction with Grb4 and modulate spine morphogenesis and synapse formation. We also reported that GIT1 tyrosine 392 is critical for PDBU-induced podosome formation by regulating PLC activation. Here, we confirm that GIT1Y321 phosphorylation is a key signal for the activation ERK $1 / 2$ and show that this signaling pathway is a major regulator of VEGF expression and fracture healing. 
The results of this study indicate that GIT1Y321 promotes VEGF release to facilitate fracture healing through the MEK/ ERK signaling pathway. These results suggest that VEGF expression through ERK1/2 signaling could be manipulated to enhance fracture healing, but the details of these signaling pathway, specifically how pY321 activates ERK and how ERK evokes VEGF release, require further study. The GIT1 is a central mediator of VEGF-regulated healing of nonunion bone fractures that may be exploited clinically in the future.

\section{Acknowledgements}

This study was supported by the National Natural and Science Foundation (81071481).

\section{References}

1. Augustin G, Antabak A, Davila S, et al: The periosteum. Part 1: anatomy, histology and molecular biology. Injury 38: 1115-1130, 2007.

2. Brandi ML and Collin-Osdoby P: Vascular biology and the skeleton. J Bone Miner Res 21: 183-192, 2006.

3. Eckardt H, Ding M, Lind M, et al: Recombinant human vascular endothelial growth factor enhances bone healing in an experimental nonunion model. J Bone Joint Surg Br 87: 1434-1438, 2005.

4. Richard DE, Berra E, Gothie E, et al: p42/44 mitogen-activated protein kinases phosphorylate hypoxia-inducible factor $1 \alpha$ (HIF-1) and enhance the transcriptional activity of HIF-1. J Biol Chem 274: 32631-32637, 1999

5. Pages G, Milanini J, Richard DE, et al: Signaling angiogenesis via p42/p44 MAP kinase cascade. J Ann NY Acad Sci 902: 187-200, 2000.

6. Witte V, Laffert B, Gintschel P, et al: Induction of HIV transcription by Nef involves Lck activation and protein kinase $\mathrm{C}$ theta raft recruitment leading to activation of ERK1/2 but not NF kappa B. J Immumol 181: 8425-8432, 2008.

7. Yin G, Haendeler J, Yan C, et al: GIT1 functions as a scaffold for MEK1-extracellular signal-regulated kinase 1 and 2 activation by angiotensin II and epidermal growth factor. Mol Cell Biol 24: 875-885, 2004

8. Pang J, Hoefen R, Pryhuber GS, et al: G-protein-coupled receptor kinase interacting protein-1 is required for pulmonary vascular development. Circulation 119: 1524-1532, 2009.

9. Turner CE, Brown MC, Perrotta JA, et al: Paxillin LD4 motif binds PAK and PIX through a novel 95-kD ankyrin repeat, ARF-GAP protein: a role in cytoskeletal remodeling. J Cell Biol 145: 851-863, 1999.

10. Yin G, Yan C and Berk BC: Ang II signaling pathways mediated by tyrosine. Int J Biochem Cell Biol 35: 780-783, 2003.

11. Webb DJ, Mayhew MW, Kovalenko M, et al: Identifucation of phosphorylation sites in GIT1. J Cell Sci 119: 2847-2850, 2006.

12. Wang J, Yin G, Menon P, et al: Phosphorylation of G proteincoupled receptor kinase 2-interacting protein 1 tyrosine 321 is required for phospholipase $\mathrm{C}$-gamma activation and podosome formation in vascular smooth muscle cells. Arterioscler Thromb Vasc Biol 30: 1976-1982, 2010.
13. Yin G, Zheng Q, Yan C, et al: GIT1 is a scaffold for ERK1/2 activation in focal adhesions. J Biol Chem 280: 27705-27712, 2005.

14. Tiscornia G, Singer O, Verma IM, et al: Production and purification of lentiviral vectors. Nat Protoc 1: 241-245, 2006.

15. Frick KK and Bushinsky DA: Chronic metabolic acidosis reversibly inhibits extracellular matrix gene expression in mouse osteoblasts. Am J Physiol Renal Physiol 275: F840-F847, 1998.

16. Sakano S, Zhu Y, Sandell L, et al: Cartilage-derived retinoic acid-sensitive protein and type II collagen expression during fracture healing are potential targets for Sox 9 regulation. J Bone Miner Res 14: 1891-1901, 1999.

17. Mayer H, Bertram H, Lindenmaier W, et al: Vascular endothelial growth factor (VEGF-A) expression in human mesenchyreal stem cells: autocrine and paracrine role on osteoblastic and endothelial differentiation. J Cell Biochem 95: 827-839, 2005.

18. Aldridge SE, Lennard TW, Williams JR and Birch MA: Vascular endothelial growth factor receptors in osteoclast diferentiation and function. Biochem Biophys Res Commun 335: 793-798, 2005.

19. Niida S, Kondo T, Hiratsuka S, et al: VEGF receptor 1 signaling is essential for osteoclast development and bone marrow formation in colony-stimulating factor 1-deficient mice. Proc Natl Acad Sci USA 102: 14016-14021, 2005.

20. Deckers MM, van Bezooijen RL, van der Horst G, et al: Bone morphogenetic proteins stimulate angiogenesis through osteoblastderived vascular endothelial growth factor A. Endocrinology 143: $1545-1553,2002$.

21. Kato K, Tokuda H, Adachi S, et al: Role of heat shock protein 27 in transforming growth factor- $\beta$ stimulated vascular endothelial growth factor release in osteoblasts. Int J Mol Med 27: 423-428, 2011.

22. Feldser D, Agani F, Iyer NV, et al: Reciprocal positive regulation of hypoxia-inducible factor 1alpha and insulin-like growth factor 2. Cancer Res 59: 3915-3918, 1999.

23. Salceda S, Beck I, Srinivas V and Caro J: Complex role of protein phosphorylation in gene activation by hypoxia. Kidney Int 51: 556-559, 1997.

24. Wu J, Brandt S and Huder SM: Lingand and cell-specific effects of signal transduction pathway inhibitors on progestin-induced vascular endothelial growth factor levels in human breast cancer cells. Mol Endocrinol 19: 312-326, 2005.

25. Hofmann KP, Scheerer P, Hildebrand PW, et al: A G proteincoupled receptor at work the rhodopsin model. Trends Biochen Sci 34: 11022-11027, 2009.

26. Claing A, Chen W, Miller, WE, et al: Beta-Arrestin-mediated ADP-ribosylation factor 6 activation and beta 2 -adrenergic receptor endocytosis. J Biol Chen 276: 42509-42513, 2001.

27. Overbaugh J, Miller AD and Eiden MV: Receptors and entry cofactors for retroviruses include single and multiple transmembrane-spanning proteins as well as newly described glycophosphatidylinositol-anchored and secreted proteins. Microbiol Mol Biol Rev 65: 371-389, 2001.

28. Fernandez J, Yaman I, Merrick WC, et al: Regulation of internal ribosome entry site-mediated translation by eukaryotic initiation factor-2alpha phosphorylation and translation of a small upstream open reading frame. J Biol Chem 277: 2050-2058, 2002.

29. Segura I, Essmann CL, Weinges S and Acker-Palmer A: Grb4 and GIT1 transduce ephrinB reversesignals modulating spine morphogenesis and synapse formation. Nat Neurosci 10: 301-310, 2007. 